\title{
Green's function retrieval and fluctuations of cross density of states in multiple scattering media
}

\author{
Julien de Rosny* \\ Institut Langevin, ESPCI, CNRS, 1 rue Vauquelin, 75231 Paris cedex 05 \\ Matthieu Davy \\ Institut d'Electronique et de Télécommunications de Rennes, University of Rennes 1, Rennes 35042, France
}

(Dated: 04/09/2013)

\begin{abstract}
In this article we derive the average and the variance of the cross-correlation of a noise wavefield. The noise cross-correlation function (NCF) is widely used to passively estimate the Green's function between two probes and is proportional to the cross density of states (CDOS) in photonic and plasmonic systems. We first explain from the ladder approximation how the diffusion halo plays the role of secondary sources to reconstruct the mean Green's function. We then show that fluctuations of NCF are governed by several non-Gaussian correlations. An infinite-range NCF correlation dominates CDOS fluctuations and proves that NCF is not a self averaging quantity with respect to the plurality of noise sources. The link between these correlations and the intensity ones is highlighted. These results are supported by numerical simulations and are of importance for passive imaging applications and material science.
\end{abstract}

A wave propagating in a multiple scattering medium is completely scrambled and generates random intensity patterns. Nevertheless, Weaver and Lobkis [1] showed in 2001 that the time derivative of the cross-correlation of an equipartitioned field measured at two positions $\mathbf{r}_{A}$ and $\mathbf{r}_{B}$ is proportionnal to the difference of the causal and anti-causal temporal Green's function (GF). This result originates from the fluctuation-dissipation theorem [2] and has provided a framework for passive imaging systems [3]. It has especially led to spectacular developments in seismology where images of the earth crust have been obtained at different scales with unprecedented resolutions [4, 5]. GF retrieval from cross-correlations of a diffuse field has also been applied to acoustic waves [6], elastic waves [7, 8, and recently electromagnetic waves [9]. The NCF has been interpreted as the field that is backpropagated by a time reversal mirror that completely surrounds a multiple scattering medium [10].

In the frequency domain, the NCF function reduces to the imaginary part of the GF, $\Im G\left(\mathbf{r}_{A}, \mathbf{r}_{B}\right)$. When the positions of the probes coincide $\left(\mathbf{r}_{A}=\mathbf{r}_{B}\right)$, the NCF linearly depends on the local density of states (LDOS) which counts the number of modes available at a given position. In optics, the LDOS determines spontaneous and stimulated emission of light. The LDOS exhibits spatial fluctuations caused by scatterers in the vicinity of the source [11 14]. The variance of the LDOS is indeed equal to the intensity correlation $C_{0}$ [12, 14, which results from local interaction. This infinite spatial range correlation was identified by Shapiro [15]. It differs from the universal intensity correlations $C_{1}, C_{2}$ and $C_{3}$. The short-range contribution $C_{1}$ simply results from Gaussian statistics. The non-Gaussian contributions $C_{2}$ and $C_{3}$ are long- and infinite-range contributions, respectively, and characterize statistics of enhanced intensity fluctuations [16.
About 10 years ago, Van Tiggelen [17] showed that in a random medium, the NCF tends to be self-averaging even though the noise sources are not equally distributed. Because multiple scattering increases the spatial diversity of the field and therefore reduces Gaussian fluctuations, the $\mathrm{NCF}$ converges more rapidly towards the average $\mathrm{NCF}$ [18. The same conclusion was derived from a parabolic approximation approach of scattering within the time reversal framework [19]. However, when the distance between $\mathbf{r}_{A}$ and $\mathbf{r}_{B}$ is larger than one elastic mean free path $\left(l_{e}\right)$, the mean GF vanishes and the self-averaging property of the NCF seems to be in contradiction with the deterministic approach that claims that the NCF is given by $\Im G\left(\mathbf{r}_{A}, \mathbf{r}_{B}\right)$. Indeed, even if $\left\|\mathbf{r}_{A}-\mathbf{r}_{B}\right\| \gg l_{e}$, $\left\langle\left|\Im G\left(\mathbf{r}_{A}, \mathbf{r}_{B}\right)\right|^{2}\right\rangle>0$. This result implies that nonGaussian correlations should contribute to NCF fluctuations.

Here we first show that the ladder approximation helps to interpret the emergence of the average NCF in a multiple scattering medium. Scatterers located in one mean free path around the probes play the role of secondary sources. Then we use a diagrammatic expansion of the diffuse field to identify the significant non-Gaussian correlations that characterize NCF fluctuations. We derive the analytical expression of the variance of the NCF for one noise source or a continuous distribution over the scattering volume. We show that the same infinite-range contribution $\gamma_{2 a}$ causes fluctuations of the cross density of states (CDOS) and explains why the NCF is not selfaveraging. We highlight why this contribution cannot be deduced from the classical intensity correlation term $C_{2}$. Finally, we shown that fluctuations of the NCF in case of a single noise source are due to non universal local terms. All those results are supported by numerical simulations.

We assume a set of uncorrelated wide-band sources of noise represented by the power spectrum function $S_{V}(\mathbf{r})$ 
distributed over a volume $V$ (or equivalently a surface in $2 \mathrm{D})$. In the frequency domain, the noise cross correlation $\zeta_{V}$ between two probes at locations $\mathbf{r}_{A}$ and $\mathbf{r}_{B}$ is given by,

$$
\zeta_{V}\left(\mathbf{r}_{A}, \mathbf{r}_{B}\right)=\int_{V} G^{*}\left(\mathbf{r}_{B}, \mathbf{r}\right) G\left(\mathbf{r}_{A}, \mathbf{r}\right) S_{V}(\mathbf{r}) d^{d} \mathbf{r}
$$

where $d$ is the dimensionality of the space (here 2 or 3 ). The frequency dependence is kept implicit. When the noise sources are uniformly distributed $\left(S_{V}(r)=S_{\infty}\right)$, the correlation of the fields is integrated over the entire scattering volume and the NCF is proportional to the imaginary part of the GF 20 ,

$$
\zeta_{\infty}\left(\mathbf{r}_{A}, \mathbf{r}_{B}\right)=-\frac{l_{a}}{k_{0}} \Im G\left(\mathbf{r}_{A}, \mathbf{r}_{B}\right) S_{\infty}
$$

Here $l_{a}$ is the absorption (inelastic) mean free path. The average value $\left\langle\zeta_{V}\right\rangle$ is governed by $\left\langle G^{*}\left(\mathbf{r}_{2}, \mathbf{r}\right) G\left(\mathbf{r}_{1}, \mathbf{r}\right)\right\rangle$. From the Bethe-Salpeter equation and the ladder approximation, $\left\langle\zeta_{V}\right\rangle$ is given by,

$$
\begin{aligned}
\left\langle\zeta_{V}\left(\mathbf{r}_{A}, \mathbf{r}_{B}\right)\right\rangle= & \int\left\langle G\left(\mathbf{r}_{B}, \mathbf{r}\right)^{*}\right\rangle\left\langle G\left(\mathbf{r}_{A}, \mathbf{r}\right)\right\rangle S_{V}(\mathbf{r}) d^{d} \mathbf{r} \\
& +\int\left\langle G\left(\mathbf{r}_{B}, \mathbf{r}^{\prime}\right)^{*}\right\rangle\left\langle G\left(\mathbf{r}_{A}, \mathbf{r}^{\prime}\right)\right\rangle F\left(\mathbf{r}^{\prime}\right) d^{d} \mathbf{r}(3)
\end{aligned}
$$

The halo function $F\left(\mathbf{r}^{\prime}\right)$ is equal to $\int \mid\left\langle\left. G\left(\mathbf{r}, \mathbf{r}^{\prime \prime}\right\rangle\right|^{2} S_{V}(\mathbf{r}) L\left(\mathbf{r}^{\prime}, \mathbf{r}^{\prime \prime}\right) d^{d} \mathbf{r}^{\prime \prime} d^{d} \mathbf{r}\right.$. The first term in Eq. (3) is the coherent contribution of the field. The second term can be interpreted using Eq. (1). The expressions are indeed similar but the power spectrum function is replaced by $F\left(\mathbf{r}^{\prime}\right)$ and the Green's functions are replaced by the mean ones. The halo that diffuses from the noise source illuminates the scatterers closer than an elastic mean free path from points A and B. Those last scattering events therefore play the role of secondary sources to build up the mean NCF.

To confirm this result, we carry out $2 \mathrm{D}$ numerical simulations in the time domain with a finite-difference timedomain (FDTD) code. The scatterers are uniformly distributed inside a ring with an inner radius of $5 \lambda_{0}\left(\lambda_{0}\right.$ is the central frequency wavelength) and an outer radius of $20 \lambda_{0}$. The mean free path is $\ell_{e}=1.5 \lambda_{0}$ and the noise is emitted from a single source outside the multiple scattering medium. The NCF $\left\langle\zeta_{V}\left(\mathbf{r}_{A}, \mathbf{r}_{B}, t\right)\right\rangle$ which is the inverse Fourier transform of Eq. (3) is recorded and averaged over 270 disorder realizations. In Fig. 1 the maps of the average NCF at different times are displayed versus $\mathbf{r}_{B}$ for a fixed position $\mathbf{r}_{A}$. We clearly observe an almost circular wavefront predicted by the coherent term in (3). A second circular wavefront focuses on point $\mathrm{A}$ at negative times and is followed by a diverging one at positive times. This contribution due to the halo appears with a skin depth of about one mean free path.
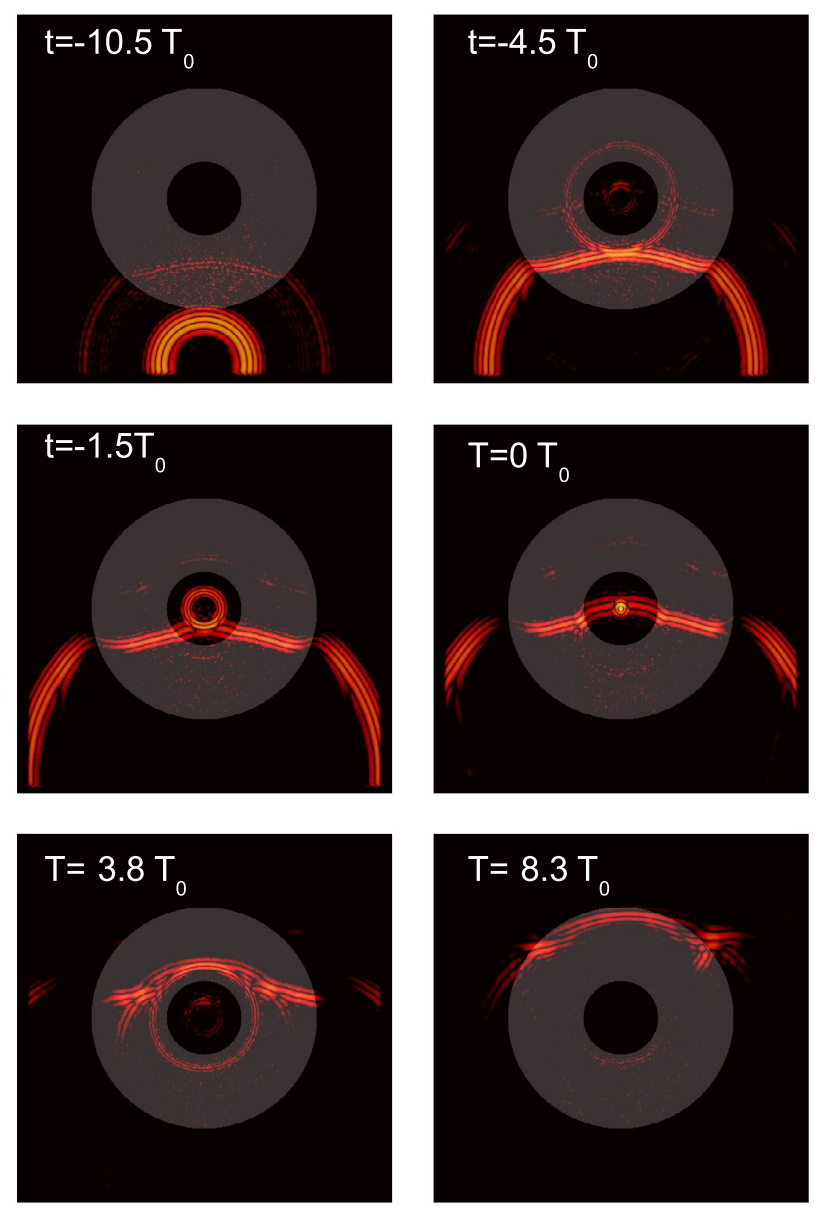

0.01

0.1 1

Figure 1. (a)-(f) Simulations of the average cross-correlated field $\left\langle\zeta_{V}\left(\mathbf{r}_{A}, \mathbf{r}_{B}, t\right)\right\rangle$ for a single noise source shown at different times. The axis are the coordinates of $\mathbf{r}_{B}$ with a $2 \mathrm{D}$ simulation domain of 50 by 50 wavelengths. The multiple scattering media is shown in gray. The single noise source and the point $\mathrm{A}$ are located respectively at the bottom of the scattering area and in the center of the figure.

The result is even more spectacular on an animation 21. Because the halo is almost uniformly distributed over at least one mean free path around $\mathbf{r}_{A},\left\langle\zeta_{V}\left(\mathbf{r}_{A}, \mathbf{r}_{B}\right)\right\rangle$ is proportional to $\left\langle G^{*}\left(\mathbf{r}_{B}, \mathbf{r}_{A}\right)\right\rangle-\left\langle G\left(\mathbf{r}_{B}, \mathbf{r}_{A}\right)\right\rangle$ because $\int\left\langle G\left(\mathbf{r}, \mathbf{r}_{A}\right)\right\rangle\left\langle G^{*}\left(\mathbf{r}, \mathbf{r}_{B}\right)\right\rangle d V=-l_{e} / k_{0} \Im\left\langle G\left(\mathbf{r}_{B}, \mathbf{r}_{A}\right)\right\rangle[22$. The mean GF (resp. conjugate mean GF) represents the diverging (resp. converging) coherent wave.

In laboratory experiments, the NCF can easily be averaged over realizations of the disorder. For instance, in optics the scatterers randomly move as a consequence of the Brownian motion. In a microwave experiments, the beads can be mixed in a toner. However, in seismology the NCF can only be measured in a single realization of the disorder. The NCF is expected to be self-averaging [18, 19] as a result of Gaussian statistics. In the context of time reversal[23] or phase-conjugation 

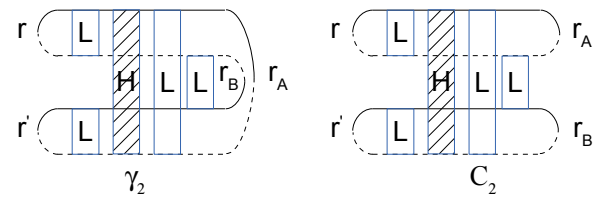

Figure 2. Comparaison between the diagram that mainly contribute to $\gamma_{2}$ and its C2 counterpart. More details can be found in supplemental material [27].

focusing [24, long range correlations that cannot be predicted by Gaussian fluctuations 25] have been observed. We show in the following how those correlations characterize the fluctuations of the $\mathrm{NCF}$ in disordered systems. To this end, we estimated the variance $\gamma$ of $\zeta_{V}\left(\mathbf{r}_{A}, \mathbf{r}_{B}\right)$. For simplicity, we have replaced $S_{V}$ by an integration over a finite volume $V$ in Eq. (1). We first consider an equipartitioned noise field, $V \rightarrow \infty$ and $\left\|\mathbf{r}_{A}-\mathbf{r}_{B}\right\| \gg l_{e}$. Equation 2 gives,

$$
\gamma=\frac{l_{a}^{2}}{2 k_{0}^{2}}\left\langle G\left(\mathbf{r}_{B}, \mathbf{r}_{A}\right) G^{*}\left(\mathbf{r}_{B}, \mathbf{r}_{A}\right)\right\rangle .
$$

This simple result shows that the NCF is not "selfaveraging" in the sense that it does not converge towards the mean GF but towards the exact GF. The NCF is therefore sensitive to scatterings that occur at a distance larger than a mean free path from the probes. This fundamental result is crucial in monitoring applications. It shows that it is possible to follow the evolution of a scatterer hidden behind a multiple scattering media[26].

In the following, we use the diagrammatic approach to interpret Eq. (4) and to address fluctuations of the NCF for a small number of sources. In the limit $\Delta r \gg \ell_{e}$, we show in the supplemental material that the main contribution to $\gamma$ is $\gamma_{2 a}$ shown in Fig. 2. Since the diagram is long-range both in $\mathbf{r}-\mathbf{r}^{\prime}$ and in $\mathbf{r}_{A}-\mathbf{r}_{B}, \gamma_{2 a}$ is of infinite range. In Fig. 2 the diagram of the long-range correlation $C_{2}$ widely used to characterize intensity fluctuations is also depicted. Even though the two diagrams look similar at first glance, we stress that $\gamma_{2 a}$ is not equal to $C_{2}$ because of an exchange between position $\mathbf{r}_{A}$ and $\mathbf{r}_{B}$ at the right side. $C_{2}$ is indeed short-range in $\mathbf{r}_{A}-\mathbf{r}_{B}$.

We show in a supplemental material [27] that the expression of $\gamma_{2 a}$ is

$$
\begin{aligned}
\gamma_{2 a}= & 2 \Delta^{4}(\Im\langle G(\mathbf{r}, \mathbf{r})\rangle)^{2} h\left|\int_{V} L(\mathbf{r}) d^{d} r\right|^{2} \\
& \times \frac{K}{D} L\left(\mathbf{r}_{B}, \mathbf{r}_{A}\right) \Im\left\langle G\left(\mathbf{r}_{A}, \mathbf{r}_{A}\right)\right\rangle \Im\left\langle G\left(\mathbf{r}_{B}, \mathbf{r}_{B}\right)\right\rangle .
\end{aligned}
$$

Here $h$ is the Hikami constant and $\Delta=l_{e} / k_{0}$. The ladder $L$ is solution of the steady state diffusion equation with absorption, i.e, $-D \nabla^{2} L(\mathbf{r})+L(\mathbf{r}) c / l_{a}=K \delta(\mathbf{r})$. For 3D samples, $h=l_{e}^{5} / 48 \pi k^{2}, K=4 \pi c / l_{e}^{2}$ and $D=l_{e} c / 3$ and for $2 \mathrm{D}$ samples, $h=l_{e}^{5} / 32 k^{3}, K=4 k_{0} / l_{e}$ and
$D=l_{e} c / 2$. In both cases, Eq. (5) yields $\gamma_{2 a}=$ $\frac{l_{a}^{2}}{2 k_{0}^{2}}\left\langle G\left(\mathbf{r}_{B}, \mathbf{r}_{A}\right) G^{*}\left(\mathbf{r}_{B}, \mathbf{r}_{A}\right)\right\rangle$. Since $\zeta_{\infty}\left(\mathbf{r}_{A}, \mathbf{r}_{B}\right)$ and CDOS are both proportional to $\Im\left\langle G\left(\mathbf{r}_{A}, \mathbf{r}_{B}\right)\right\rangle$, CDOS fluctuations also result from the same infinite-range term $\gamma_{2 a}$.

The expansion of the Hikami vertex given in the SI that leads to Eq. (5) is valid only for $\left\|\mathbf{r}_{A}-\mathbf{r}_{B}\right\|<l_{e}$. Nevertheless in the case of coinciding probes $\mathbf{r}_{B}=\mathbf{r}_{A}$, the NCF becomes proportional to the LDOS. NCF fluctuations are then characterized by the correlation $\gamma_{0 a}$ which is proportional to the $C_{0}$ intensity correlation [12, 14]. This non-gaussian term depends on the details of the local disorder around the probe and involves a non-universal vertex $\chi_{0}$ [15], such as $\gamma_{0 a}=$ $2 \delta V^{2} \Delta^{2}(\Im\langle G(\mathbf{r}, \mathbf{r})\rangle)^{2}\left|\int_{V} L(\mathbf{r}) d^{3} r\right|^{2} \chi_{0} \cdot \gamma_{0 a}$ and $\gamma_{2 a}$ can be seen as the two asymptotic regimes $\Delta r \ll \ell_{e}$ and $\Delta r \gg \ell_{e}$, respectively, of the variance $\gamma$ which characterizes the non-Gaussian fluctuations of the NCF in mesoscopic multiple scattering media.

We perform numerical simulations to support these derivations. The $2 \mathrm{D}$ multiple scattering medium is made of $10^{4}$ isotropic scatterers enclosed in a disk of diameter $100 \lambda_{0}$. Here, $l_{a} \sim 63 \lambda_{0}$ and $l_{e} \sim 2 \lambda_{0}$. Those parameters ensure that the system is in the diffusive regime. The sample is illuminated from $N$ independent noise sources embedded in the medium. The NCF is computed from a scattering matrix inversion method. For a single disorder realization, the NCF is seen in Fig. 3(a) to converge towards $\Im\left\langle G\left(\mathbf{r}_{A}, \mathbf{r}_{B}\right)\right\rangle$ for $N=10^{4}$. On Fig. $3(\mathrm{~b})$ we observe that $\gamma$ is maximum for $\Delta r=0$ due to the $\gamma_{0 a}$ contribution and then falls rapidly with $\Delta r$ until $\gamma_{0 a}$ vanishes for $\Delta r \sim \lambda_{0} / 2$. For $\Delta r \gg \ell_{e}, \gamma$ decreases exponentially because of losses in the medium. Simulations are in very good agreement with Eq. (5) in which the ladder $L$ is solution of the $2 \mathrm{D}$ diffusion equation with losses, $L(\Delta r)=\beta K_{0}\left(\Delta r \sqrt{c / D l_{a}}\right) / 2 \pi D$. This confirms that $\gamma_{2 a}$ is of infinite-range.

The condition of an isotropic distribution of noise sources is most of the time not fulfilled in seismology and in acoustics. For $\Delta r \gg \ell_{e}, \gamma$ is seen in Fig. 4 4 to increase as $N^{2}$ for $N>200$. In this range, the NCF is close to $\Im\left\langle G\left(\mathbf{r}_{A}, \mathbf{r}_{B}\right)\right\rangle$ in Fig. 3(a) and the non-Gaussian contribution $\gamma_{2 a}$ (which scales as $N^{2}$ ) overcomes the $\gamma_{1}$ Gaussian contribution (C1-like contribution, see supplemental matetial) which scales as $N$ [18. Finally, $\gamma$ saturates for $N \sim 10^{4}$ since the sample is already illuminated uniformly. Moreover $\gamma$ is larger than $\gamma_{1}$ for $N \sim 1$. This indicates that in addition to $\gamma_{1}$ other diagrams are contributing to $\gamma$. Interference indeed occurs in the vicinity of the noise source location $\mathbf{r}_{S}$ and another $C_{0}$-like correlation has to be taken into account. Its expression is given by $2 \delta V^{2} \Delta^{2} L\left(\mathbf{r}_{B}, \mathbf{r}_{S}\right) L\left(\mathbf{r}_{A}, \mathbf{r}_{S}\right) \Im\left\langle G\left(\mathbf{r}_{A}, \mathbf{r}_{A}\right)\right\rangle \Im\left\langle G\left(\mathbf{r}_{B}, \mathbf{r}_{B}\right)\right\rangle \chi_{0}$. The volume $\delta V$ of the single source is assumed smaller than $l_{e}^{3}$. We finally note that in the case of $\Delta r \ll l_{e}$ and a single noise source, $\gamma$ is 

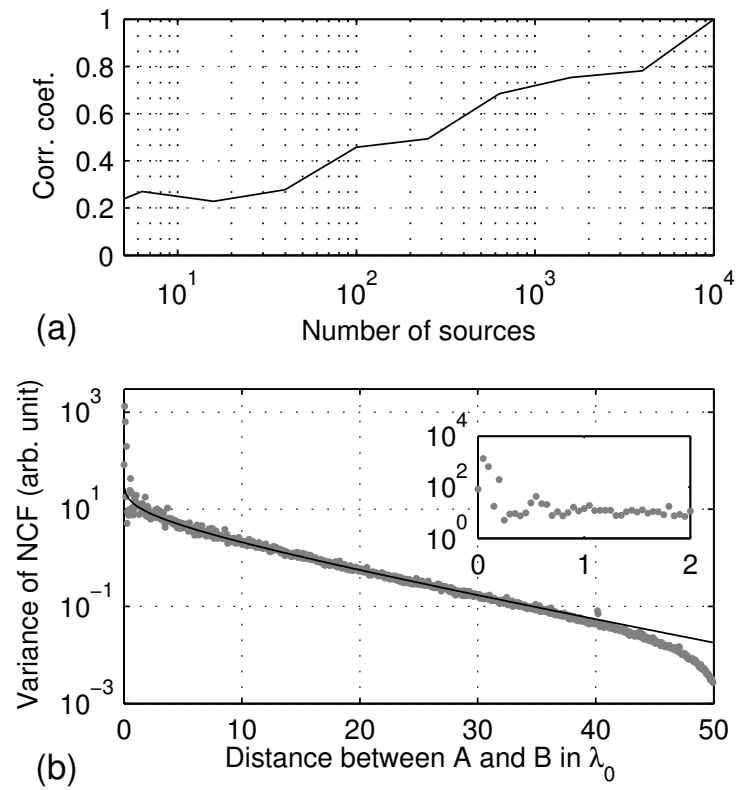

Figure 3. (a) Evolution with $N$ of the normalized correlation coefficient between $\Im\left\langle G\left(\mathbf{r}_{A}, \mathbf{r}_{B}\right)\right\rangle$ and $\zeta$ for a single realization of disorder. (b) Variance of $\zeta_{\infty}\left(\mathbf{r}_{A}, \mathbf{r}_{B}\right)\left(N=10^{4}\right)$ with $\Delta r$ estimated from the averaging over 500 disorder realizations. The black line is given by Eq. (5).

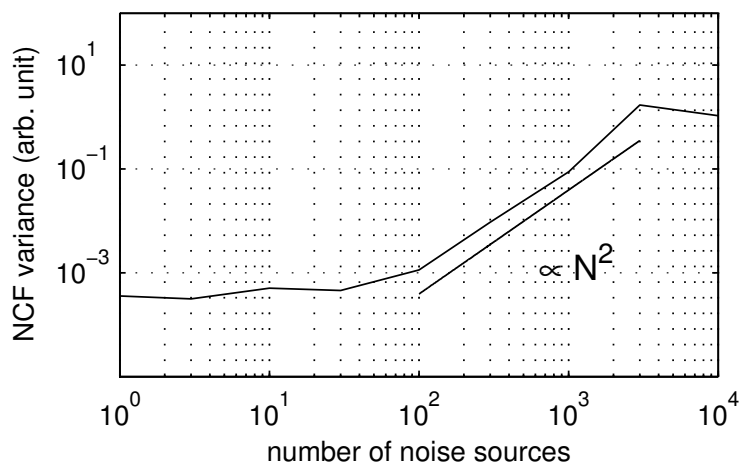

Figure 4. Simulations of the variance of the NCF with $N$ for $\Delta r \sim 15 \ell_{e}$ obtained with $5 \mathrm{e} 5$ disorder configurations.

given by the sum of two $C_{0}$ contributions [28, $\gamma=$ $2 \delta V^{2} \Delta^{2}\left|L\left(\mathbf{r}_{A}, \mathbf{r}_{S}\right)\right|^{2} \chi_{0}\left(\Im\left\langle G\left(\mathbf{r}_{A}, \mathbf{r}_{A}\right)\right\rangle^{2}+\Im\left\langle G\left(\mathbf{r}_{S}, \mathbf{r}_{S}\right)\right\rangle^{2}\right)$. Those considerations confirm the intuitive result that fluctuations of NCF caused by a source located inside a multiple scattering medium are stronger than fluctuations caused by a source outside this disordered medium which only involves Gaussian fluctuations.

In conclusion, we have used the multiple scattering theory to demonstrate the role of scatterers in the retrieval of the GF and to interpret fluctuations of the NCF in terms of diffuse light interference. To that end, we introduced an original diagrammatic contributions. Those fundamental results can be applied to many different fields such as seismology, acoustics, microwave, optics or material science. In acoustics and in seismology, the estimation of the NCF is easily performed by the direct crosscorrelation of recorded time-depend fields. However, the noise sources are usually not uniformly distributed and a generalization of our approach to more complex source distributions would be a probe of the convergence of the NCF towards the GF for a single realization of disorder. This issue is of importance for imaging purposes. On the other hand, in optics, one can take benefit of the thermal noise that is uniform at thermal equilibrium. But then it is more tedious to measure the NCF. In material science, metallic nanostructures can for instance be excited with surface plasmons in disordered media. Measuring the $\mathrm{NCF}$ at thermal equilibrium would make possible to estimate the CDOS. We suggest the experiment consisting in the measurement of the fluctuations of the field intensity diffracted by two tips on a metallic surface at thermal equilibrium where plasmons are multiply scattered to estimate $\gamma_{2}$ fluctuations. This would be an extension of thermal radiation scanning tunneling microscopy [29].

We wish to thank Roger Maynard, Philippe Roux, Remi Carminati and Boris Shapiro for fruitful discussions. This work have been partially supported by ANR grant ANR-10-BLAN-0124 OPTRANS.

* julien.derosny@espci.fr

[1] R. L. Weaver and O. I. Lobkis, Phys. Rev. Lett. 87, 134301 (SEP 24 2001), ISSN 0031-9007

[2] H. Callen and T. Welton, Phys. Rev. 83, 34 (1951), ISSN 0031-899X

[3] K. Wapenaar, E. Slob, and R. Snieder, Phys. Rev. Lett. 97, 234301 (DEC 8 2006), ISSN 0031-9007

[4] M. Campillo and A. Paul, Science 299, 547 (JAN 24 2003), ISSN 0036-8075

[5] N. M. Shapiro, M. Campillo, L. Stehly, and M. H. Ritzwoller, Science 307, 1615 (MAR 11 2005), ISSN 00368075

[6] P. Roux, W. Kuperman, and N. Grp, J. Acoust. Soc. Am. 116, 1995 (OCT 2004), ISSN 0001-4966

[7] R. L. Weaver and O. I. Lobkis, J. Acoust. Soc. Am. 113, 2611 (2003)

[8] K. Wapenaar, Phys. Rev. Lett. 93, 254301 (DEC 17 2004), ISSN 0031-9007

[9] M. Davy, M. Fink, and J. de Rosny, Phys. Rev. Lett. 110, 203901 (2013), http://prl.aps.org/abstract/ PRL/v110/i20/e203901

[10] A. Derode, E. Larose, M. Tanter, J. de Rosny, A. Tourin, M. Campillo, and M. Fink, J. Acoust. Soc. Am. 113, 2973 (JUN 2003), ISSN 0001-4966

[11] A. Mirlin, Phys. Rep. 326, 259 (2000), http: //www.sciencedirect.com/science/article/pii/ S0370157399000915

[12] B. Van Tiggelen and S. Skipetrov, Phys. Rev. E 73, 045601 (2006), http://pre.aps.org/abstract/PRE/ v73/i4/e045601

[13] M. D. Birowosuto, S. E. Skipetrov, W. L. Vos, and A. P. 
Mosk, Phys. Rev. Lett. 105, 013904 (2010)

[14] A. Cazé, R. Pierrat, and R. Carminati, Phys. Rev. A 82, 043823 (Oct 2010), http://link.aps.org/doi/10. 1103/PhysRevA . 82.043823

[15] B. Shapiro, Phys. Rev. Lett. 83, 4733 (1999), http:// link.aps.org/doi/10.1103/PhysRevLett.83.4733

[16] E. Akkermans and G. Montambaux, Mesoscopic physics of electrons and photons (Cambridge University Press, 2007)

[17] B. van Tiggelen, Phys. Rev. Lett. 91, 243904 (DEC 12 2003), ISSN 0031-9007

[18] E. Larose, L. Margerin, A. Derode, B. van Tiggelen, M. Campillo, N. Shapiro, A. Paul, L. Stehly, and M. Tanter, Geophys. 71, SI11 (2006)

[19] G. Bal, G. Papanicolaou, and L. Ryzhik, Stoch. Dyn. 2, 507 (2002), http://www.worldscientific.com/doi/ abs/10.1142/S0219493702000522

[20] R. L. Weaver and O. I. Lobkis, J. Acoust. Soc. Am. 116, 2731 (2004), http://link.aip.org/link/?JASMAN/116/ $2731 / 1$

[21] J. de Rosny and M. Davy, "Movie of the cross-correlated mean field," Electronic (Oct. 2013), http://ftp.espci. $\mathrm{fr} / \mathrm{prl}$ _movie_rosny_davy/movie.avi

[22] This relation [16] is similar to Eq. (2) but the elastic mean free path replaces the absorption mean free path because the attenuation of mean field is mainly due to elastic scattering.

[23] A. Derode, E. Larose, M. Campillo, and M. Fink, Appl. Phys. Lett. 83, 3054 (OCT 13 2003), ISSN 0003-6951

[24] M. Davy, Z. Shi, and A. Z. Genack, Phys. Rev. B 85, 035105 (2012)

[25] The variance of a self-averaging quantity should fall towards 0 when the quantity is integrated over time or space.

[26] L. Bonneau, C. Prada, M. Fink, and A. Tourin, Waves in Random and Complex Media 22, 109 (2012), http://www .tandfonline.com/doi/abs/10.1080/ 17455030.2011 .630760

[27] J. de Rosny and M. Davy, "Supplementary material : full derivation of the $\gamma_{2 a}$ contribution,"

[28] A. Retzker and B. Shapiro, Pramana 58, 225 (2002), http://link.springer.com/article/10.1007/ s12043-002-0009- $\mathrm{x}$

[29] Y. De Wilde, F. Formanek, R. Carminati, B. Gralak, P.-A. Lemoine, K. Joulain, J.-P. Mulet, Y. Chen, and J.-J. Greffet, Nature 444, 740 (2006), http://www.nature.com/nature/journal/vaop/ ncurrent/full/nature05265.html 